\title{
COUNTS OF AIR-BORNE BACTERIA AS INDICES OF AIR HYGIENE
}

\author{
BY D. D. REID \\ Department of Medical Statistics and Epidemiology, London School of \\ Hygiene and Tropical Medicine \\ O. M. LIDWELL AND R. E. O. WILLIAMS \\ Air Hygiene Laboratory, Central Public Health Laboratory, London
}

(With 1 Figure in the Text)

The number of bacteria in air samples taken in schoolrooms can be, to some extent, related to changes in the environment or to actions of the occupants (Williams, Lidwell \& Hirch, 1956). The concentration of Streptococcus salivarius in the air, for example, rises with increase in the amount of talking and, to a much less extent, with increase of activity. The count of Str. salivarius may thus be taken as a reasonable index of the degree of recent bacterial pollution of the room air by material from the mouth and respiratory tracts of the occupants. Counts of the general bacterial flora, on the other hand, appear to reflect dispersal of organisms from clothing and floor dust.

Implicit in these findings is the suggestion that counts of air-borne bacteria might be used as indices of the hygienic state of the air of inhabited rooms. In this paper we attempt to find out whether the number of bacteria found in the air of school classrooms is significantly related to the incidence and cross-infection risks of disease among the children. Only if such a relation exists can the counts of air-borne bacteria be considered as potential indices of air hygiene.

\section{MATERIAL AND METHODS \\ Sources of data}

In a three-year trial of air disinfection by ultra-violet irradiation already described in detail (Air Hygiene Committee, 1954; Williams et al. 1956), two randomly selected classrooms were visited each day and samples of air were examined for enumeration of air-borne bacteria. Records were also kept of temperature, relative humidity and ventilation rates indoors and weather conditions out of doors at the same time.

The class teachers recorded all absences of children from school, irrespective of cause, lasting one whole day or more. The reasons for absences given by the mother or the family doctor were classified according to the International Classification of Diseases, Injuries and Causes of Death (World Health Organization, 1948); in cases of doubt, health visitors made further inquiries at the home. For many short absences the inevitably vague descriptions made classification difficult. In this paper we have included 'chest colds' lasting less than 7 days under the acute colds, 
while 'sore throats' are grouped under 'tonsillitis and other acute throat affections'. Syndromes where alimentary upset accompanied symptoms of respiratory infection were grouped, together with those labelled as 'gastric 'flu', with all the other gastro-intestinal disorders.

Incidence rates, expressed as fresh absences per 100 child-years, were calculated on the basis of the mid-term class or school-department populations.

\section{Methods of analysis}

In the case of certain specific infections the risk of cross-infection can be estimated from classroom epidemics. With less well-defined syndromes, which comprise most of the causes of sickness absence, this is impossible and the only measure available is the incidence of absence.

Measles. Of the specific diseases, only measles was sufficiently prevalent during the period of study for comparative estimates of cross-infection in relation to air contamination to be made. Measles has for this purpose several advantages: diagnosis is reasonably precise; all the schools in a district are affected at much the same time; the first date of absence is precisely known, and immunity can usually be inferred from a history of a previous attack. The detailed analysis of these measles outbreaks has already been described (Air Hygiene Committee, 1954): the risk of cross-infection in different environmental circumstances was measured by the secondary attack rate expressed as the percentage of susceptible children attacked within 7-16 days after the introduction of a primary case of measles into the classroom.

Actual measurements of the environmental conditions within a particular classroom were rarely made during the 3 days before the first day of absence of a primary case; we have, therefore, used as measures of the conditions prevailing during these 3 days two alternative estimates:

(1) The mean values of the indoor temperature, indoor relative humidity and the ventilation rate, derived from all the classroom visits to all the schools during the 3 days. These means give a measure of the weather conditions, as modified by indoor heating and ventilation, existing during the period of presumed maximum infectivity of the primary case.

(2) The mean values of the same variables, together with the levels of talking, activity and bacterial air count, averaged over all the observations made in the particular classroom during the whole of that school term. This we used as an estimate of the classroom characteristics that might have affected the air-borne spread of infection; its efficiency as a measure of these characteristics depends upon reasonable consistency in the behaviour of the class and in the teacher's views on suitable room temperatures and ventilation.

Talking and activity in a classroom are closely related to the age of the children; the younger children are usually the most active and the most talkative. Since susceptibility to infection is also related to age, we have included the mean age of the class as a major variable in the calculations.

Other causes of absence. For most diseases, no cross-infection rates could be calculated and we have had to use incidence rates. Four groups of the most 
common disorders were studied, namely, acute nasopharyngitis, ear affections, sore throat and tonsillitis, and the group of gastric disturbances. Absences due to accidents were also included in the analysis as a control against spurious correlations.

The data comprising both incidence rates and the environmental measurements were grouped for analysis in two ways: averages for particular classrooms for an 'epidemic year' (September-July), or averages over all classrooms for each of the thirty-four 4-weekly periods of the study. In the first mode of analysis the major differences in sickness experience and classroom behaviour reflect differences in the age of the children; in the second, the period-to-period changes are inevitably dominated by seasonal changes. Some adjustment for these major sources of variation was made by including the mean age of class in the first analysis and solar radiation in the second.

Where appropriate, an angular transformation was used for the incidence rates; the counts of bacteria were recorded in logarithmic form. Product moment correlation coefficients were calculated between the various measures of disease incidence, bacterial count and environmental conditions used in the survey. Partial correlation analysis was then employed to determine the degree of relationship, e.g. between the measles secondary attack rate and the Str. salivarius count remaining after the effects of variations in other relevant factors such as talking and humidity had been taken into account.

\section{RESULTS}

In Table 1 are shown the correlations between the secondary attack rate for measles following classroom introductions of infection and the various environmental measurements. Only the correlations with the count of Str. salivarius consistently reach significance, and they are little affected by adjusting for simultaneous variations in the other characteristics included in the analysis. The Str. salivarius count was greatly reduced by the irradiation, but it is clear from the table that its correlation with the measles secondary attack rate was not simply a difference between the irradiated and control schools since the correlations were similar in the unirradiated schools. The results are shown graphically in Fig. 1. In classrooms with generally low counts of Str. salivarius the average secondary attack rate for measles was also low-below $10 \%$. In those rooms where the count was higher than about 0.25 colony/cu.ft., however, the measles secondary attack rate was also higher, being above $60 \%$ when the count approached $1 \cdot 0 /$ cu.ft.

There is a negative correlation, near the limit of significance, with the relative humidity at about the time of infection. Measles epidemics commonly occur in the spring, which is the period of low indoor relative humidity.

The correlation coefficients between the incidence of the five common causes of absence and the environmental factors are shown in Tables 2 and 3 . It is clear that, after age and seasonal differences have been discounted, accidents have no particular association with any of the variables under consideration. The classroom analysis shows a group of high coefficients against the count of general flora (see Table 3, col. 3); that is, there was a higher incidence of the diseases (other than 


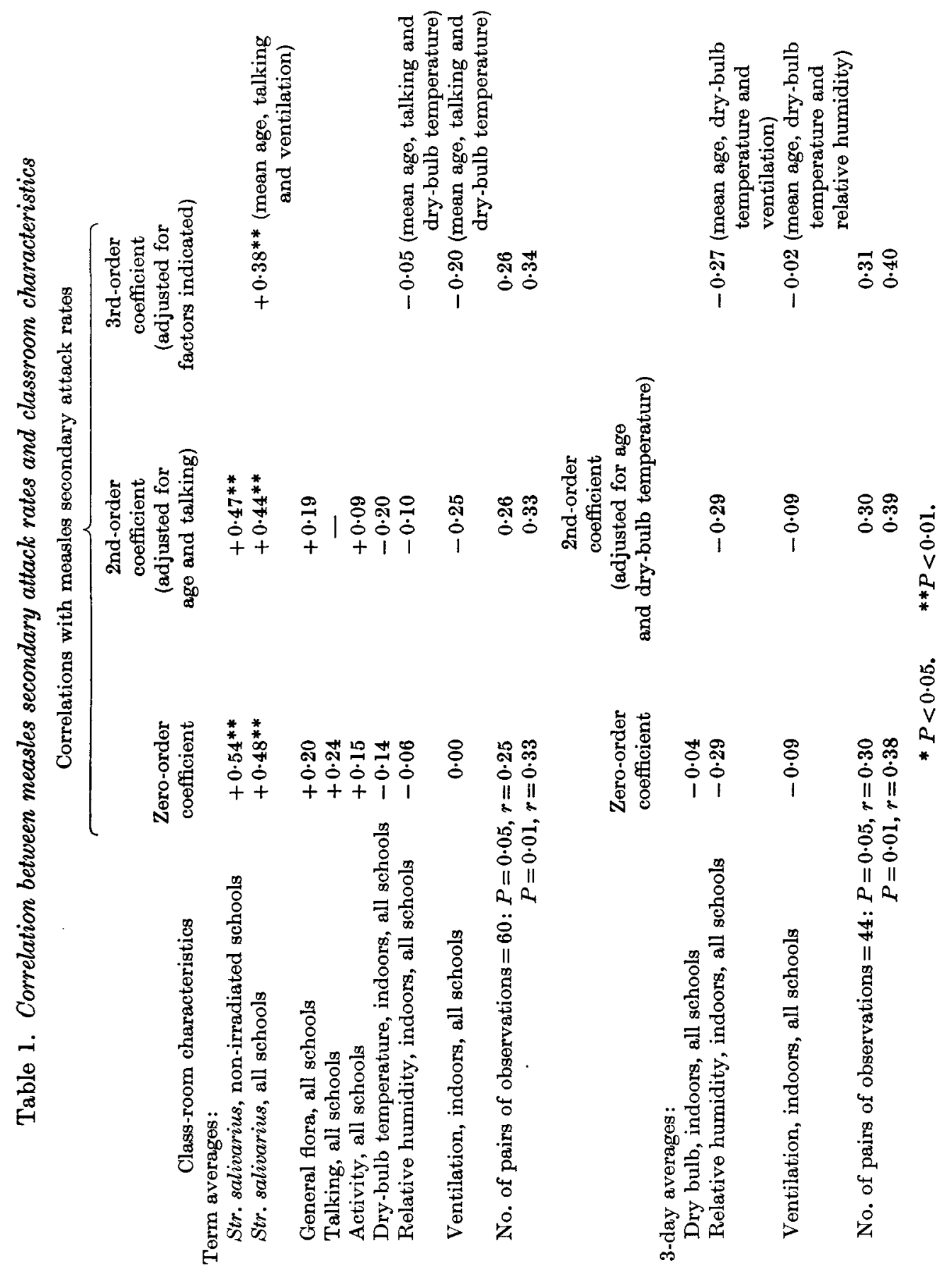




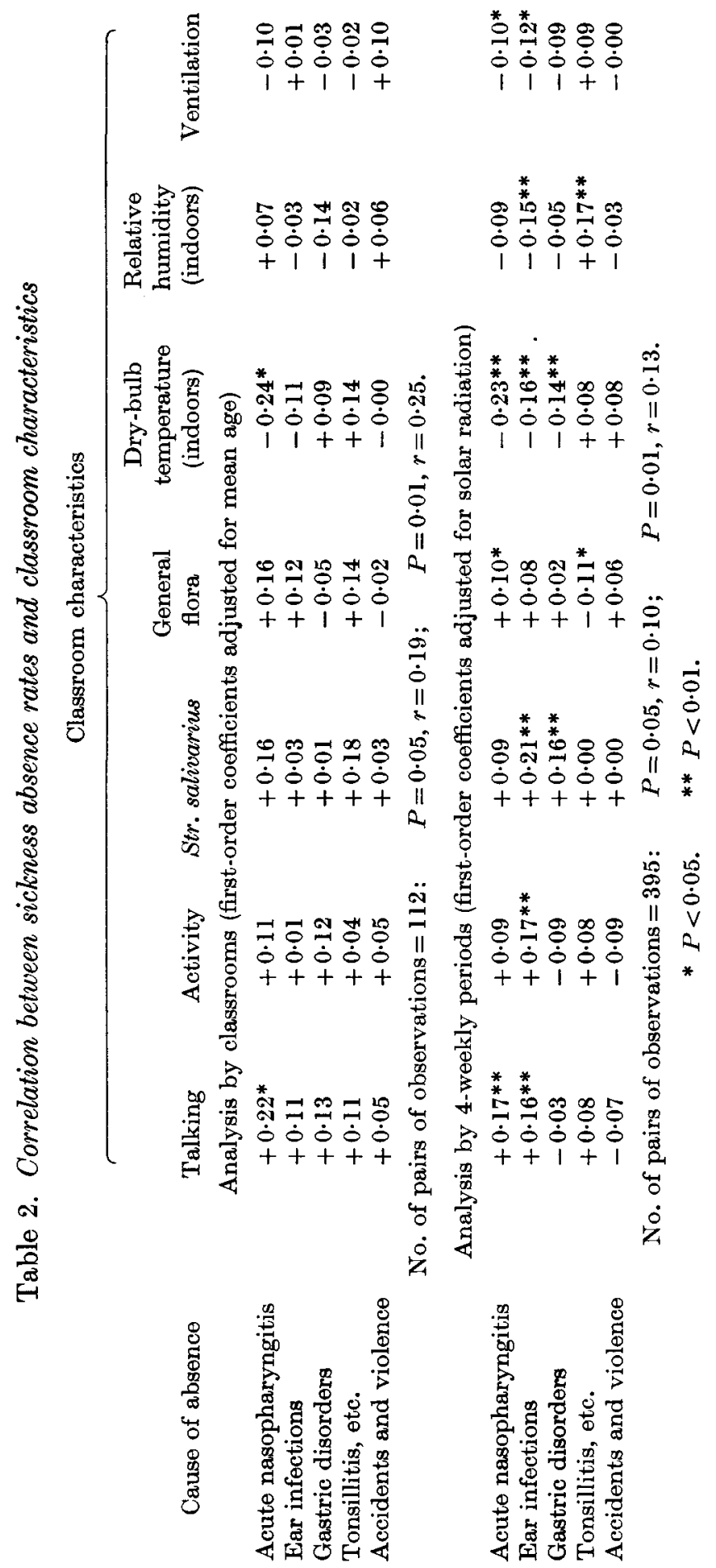




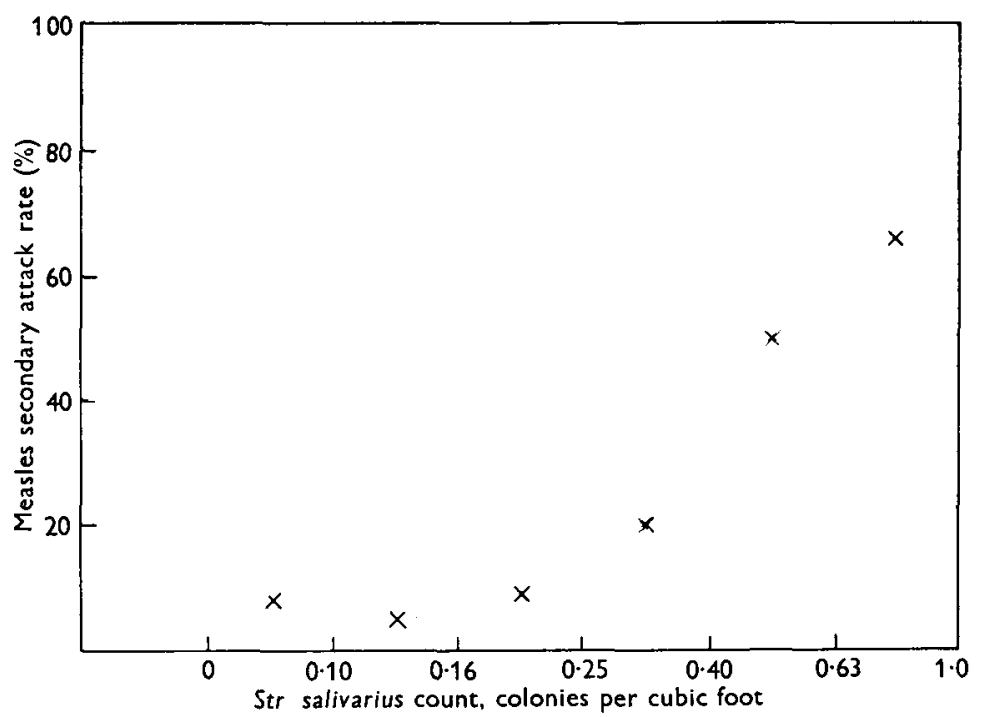

Fig. 1. Counts of air-borne Str. salivarius and measles cross-infection rates in non-irradiated classrooms.

Table 3. Correlation between sickness absence rates and classroom characteristics

\begin{tabular}{|c|c|c|c|c|c|}
\hline & & & $m$ charact & & \\
\hline Cause of absence & Activity & $\begin{array}{c}\text { Str. } \\
\text { salivarius }\end{array}$ & $\begin{array}{c}\text { General } \\
\text { flora }\end{array}$ & $\begin{array}{l}\text { Relative } \\
\text { humidity } \\
\text { (indoors) }\end{array}$ & Ventilation \\
\hline
\end{tabular}

Analysis by classroom

(third-order coefficients adjusted for mean age of class, dry-bulb temperature indoors and talking)

$\begin{array}{llcccr}\text { Acute nasopharyngitis } & +0.07 & +0.08 & +0.40^{* *} & +0.03 & -0.04 \\ \text { Ear infections } & -0.04 & -0.03 & +0.29^{* *} & -0.05 & +0.04 \\ \text { Gastric disorders } & +0.12 & +0.00 & -0.01 & -0.12 & -0.00 \\ \text { Tonsillitis, etc. } & -0.07 & +0.16 & +0.42^{* *} & +0.01 & -0.06\end{array}$

No. of pairs of observations $=112: \quad P=0.05, r=0.19 ; \quad P=0.01, r=0.25$

Analysis by 4-weekly periods (third-order coefficients adjusted for solar radiation, dry-bulb temperature indoors and talking)

$\begin{array}{llllll}\text { Acute nasopharyngitis } & -0.01 & +0.01 & +0.03 & -0.07 & -0.06 \\ \text { Ear infections } & +0.11^{*} & +0.15^{* *} & +0.02 & -0.14^{* *} & -0.09 \\ \text { Gastric disorders } & -0.09 & +0.17^{* *} & +0.02 & -0.04 & -0.07 \\ \text { Tonsillitis, etc. } & +0.03 & -0.02 & -0.14^{* *} & +0.16^{* *} & +0.08\end{array}$

No. of pairs of observations $=395: \quad P=0.05, r=0.10 ; \quad P=0 \cdot 01, r=0 \cdot 13$

$* P<0.05$. ** $P<0.01$. 
gastric disorders) in those classrooms where, under comparable conditions of temperature and talking, and with children of similar age, there was a greater dispersal of organisms from secondary sources. Similar correlations were not found in the analysis by 4-weekly periods, and, indeed, the correlation between tonsillitis and general flora is here negative. There are some apparently significant, but small, correlations in the analysis by 4-weekly periods between the Str. salivarius count and the incidence of ear infections and of gastric disorders and between the inside relative humidity and the incidence of ear infections and of tonsillitis. It is, however, possible that this analysis is still confused by seasonal or other effects not entirely accounted for by variations in solar radiation and temperature.

\section{DISCUSSION}

The background to these investigations was the suggestion that some count of the numbers of bacteria, especially streptococci, found in the air of occupied places, might be used to indicate the hygienic state of the air in the same way as the Escherischia coli test is used to indicate the purity of drinking water supplies.

With the exception of the work of Carnelly, Haldane \& Anderson (1887), little has been done to relate the incidence of human disease in inhabited spaces to objective measures of the pollution of the air by material which may come from human sources. In these early studies, the concentrations of carbonic acid, organic matters and the total number of micro-organisms were measured in houses, schools and factories in Dundee. It was shown that concentrations of these air pollutants were higher in overcrowded conditions and that the mortality rates in the city were also higher in overcrowded homes. There was, however, no direct comparison made in particular homes or schools between the morbidity of the occupants and the degree of pollution by bacteria derived from the respiratory tract; and we know of no other similar epidemiological studies of human infections occurring in normal conditions which might justify the use of counts of air-borne bacteria as a hygienic index.

The coli test for water depends on a presumed correlation between the incidence of disease and the presence of 'indicator organisms' - not themselves pathogenicin the water. This presumption rests on the facts that the human intestinal canal is a constant and the only significant source of the 'typical' Esch. coli and that enteric diseases are certainly spread by water polluted with human excrement. It may be noted, however, that even with this long-established test there is very little direct confirmation of its validity in the form of correlations between the index of water pollution and the incidence of water-borne disease.

The comparable postulates for the employment of the streptococcal count in air would be that the streptococci are found constantly and only as respiratory commensals, and that respiratory disease is known to be transmitted in the air space sampled by air-borne particles derived from the respiratory tract.

Str. salivarius probably meets the first of these requirements, but too little is known about the exact mode of transmission of respiratory illness for us to act on the second assumption. For the bacterial count to be affirmed as an index of air 
hygiene we must show a direct correlation between the count and the risk of transfer of infection in the environment tested. In considering correlations between bacterial count and disease there is, however, an important reservation. The bacterial count in the air depends on particular activities which may in turn be correlated with some other unknown factor-an action of the occupants or an environmental condition - that leads to spread of the disease. The correlation may still indicate that the count is a valid measure of the risk of disease transfer in the particular rooms observed; but it may not be so elsewhere where the unknown factor varies independently.

The Str. salivarius counts in the classroom air certainly seemed to indicate the risk of transfer of measles; this would support the idea that measles is truly an 'air-borne' disease. But the correlation does not distinguish between spread by large droplets and spread by small droplets-for the activities that generated the small particles collected in our air sampling inevitably also generated large particles which might equally well carry infection between the children. On the other hand, ultra-violet irradiation of the upper air, which could have affected only the smaller widely circulating particles, appreciably reduced both the Str. salivarius count and the secondary attack rate. It seems likely, therefore, that the correlation observed between count and measles transfer is a direct relationship and not due to a coincidental variation in a third factor.

Measles is the only disease for which we have been able to get any direct measure of transfer within the classrooms. For the other diseases we have had to rely on incidence rates which must also derive from infections acquired in other places and can at best give only a rough indication of the risks of schoolroom crossinfection.

The correlations observed between the general flora counts and various respiratory tract affections are more difficult to explain than those for measles. The only disorders apparently affected by ultra-violet irradiation were sore throats and ear affections; and both these diseases are sometimes due to haemolytic streptococciorganisms which are certainly killed by irradiation. We can only suggest that some of these two types of respiratory illness result from infection with widely circulating particles of irradiation-sensitive haemolytic streptococci. On the other hand, the correlation of the other undifferentiated respiratory affections with the general flora count and not with the Str. salivarius count could arise in several ways. The pathogens may derive from parts of the respiratory tract, such as the nose, which contain no Str. salivarius; they may survive longer on clothing than Str. salivarius; or they may be transferred by actions which disseminate general flora and at the same time favour direct droplet spray infection rather than wide circulation in droplet nuclei. However unlikely suggestions, such as the second, may be, they serve to underline the clear distinction that should be made between the hygienic indications of Str. salivarius counts in relation to measles and of the general flora count in relation to non-specific respiratory affections. In the first instance, the association is a reasonably direct one because the indicator organisms from the respiratory tract appear to behave like the air-borne pathogens concerned. In the second case, the association is obviously quite indirect and the results of this 
analysis would not justify the use of the general flora count as an index of roominfection risk.

The correlations shown in the analysis by 4-week periods are still less easily explained. The most plausible suggestion would seem to be that they derive indirectly from seasonal or other secular changes.

We may conclude that the Str. salivarius count shows promise of utility as an index of air hygiene in certain limited circumstances but that the significance of the correlation between general flora counts and respiratory disease can be further assessed only by experiments designed for that purpose. Meanwhile, the value of air counts of the general flora as a measure of the healthiness of the environment must be considered uncertain.

\section{SUMMARY AND CONCLUSIONS}

An attempt has been made to assess the value of counts of bacteria in the air of occupied rooms as indices of the risks of air-borne cross-infection in these rooms. Data collected during a field trial of ultra-violet irradiation in schoolrooms have been analysed to establish the correlation between counts of Streptococcus salivarius or of the general flora and classroom secondary attack rates for measles and the incidence of four groups of respiratory affections. Variations in classroom environment and child age and behaviour were taken into account in the analysis.

The Str. salivarius count has been shown to be significantly related to the risk of classroom transfer of measles, but not to the incidence of respiratory disease. For the general flora counts the reverse was true.

A discussion of these results in the light of the effects of ultra-violet air disinfection on the incidence of presumably air-borne diseases leads to the conclusion that the Str. salivarius count may reasonably be taken as index of the risk of classroom transfer of measles, but that no such assumption is possible about the value of general flora counts in the measurement of air hygiene in relation to respiratory diseases.

We are particularly indebted to Mrs B. M. Hunt and Miss D. Bobby for carrying out the heavy computing involved in this study and to Mrs B.S. King for secretarial help and the drawing of Fig. 1.

\section{REFERENCES}

Air Hygiene Committee (1954). Air disinfection with ultra-violet irradiation. Spec. Rep. Ser. med. Res. Coun., Lond., no. 283.

Carnelly, T., Haldane, J. S. \& Anderson, A. M. (1887). The carbonic acid, organic matter, and micro-organisms in air, more especially of dwellings and schools. Phil. Trans. B, 178, 61.

Williams, R. E. O., LiDWell, O. M. \& Hirch, ANN (1956). The bacterial flora of the air of occupied rooms. J. Hyg., Camb., 54, 512.

World Health Organization (1948). Manual of the International Classification of Diseases, Injuries and Causes of Death. Geneva: W.H.O. 